STOMACH

\title{
Differences in the sensorimotor response to distension between the proximal and distal stomach in humans
}

\author{
K-J Lee, R Vos, J Janssens, J Tack
}

Gut 2004;53:938-943. doi: 10.1136/gut.2003.025031

Background: It is not known which region of the stomach is responsible for symptom generation or whether symptoms induced by gastric distension are region specific. Also, it is unclear whether low level gastric distension has a modulatory role on gastric tone and mechanosensitivity.

Aims: To define differences in the sensorimotor response to distension between proximal and distal gastric distension, and to determine the effects of low level gastric distension on gastric tone and mechanosensitivity.

See end of article for authors' affiliations

Methods: In 14 healthy volunteers, a double barostat assembly incorporating a distal (antral) and proximal (fundic) bag was introduced into the stomach. Pressure sensitivity tests with either bag were performed with and without simultaneous background distension of the other bag in a randomised

Correspondence to: Dr J Tack, Division of Gastroenterology,

Department of Internal Medicine, University Hospital Gasthuisberg, Herestraat 49, B-3000 Leuven, Belgium; Jan.Tack@

med.kuleuven.ac.be manner. Proximal gastric accommodation to a meal was measured with and without simultaneous distal gastric distension.

Results: The distal stomach was less compliant than the proximal stomach to low level distension. Thresholds for first perception and discomfort, and symptom profiles did not differ between distal and proximal gastric distension. Simultaneously applied low level gastric distension of one segment did not affect gastric mechanosensitivity of the other segment. Both the proximal and distal stomach relax after ingestion of a meal. Simultaneous low level antral distension decreases proximal gastric accommodation to a meal.

Accepted for publication Conclusions: Compared with the proximal stomach, the distal stomach is less compliant but its mechanosensitivity is not different. Symptoms induced by gastric distension are not region specific and no spatial summation occurred. Meal induced relaxation occurs both in the proximal and distal stomach.

$\mathrm{T}$ he different roles of the proximal and distal stomach in the digestive process are well recognised. The proximal stomach accommodates food through receptive relaxation and regulates its transfer to the distal stomach. In contrast, the distal stomach is involved in grinding and mixing of intragastric contents and regulates nutrient delivery to the duodenum. ${ }^{1}$ After ingestion, nutrients are normally located predominantly in the proximal stomach, with progressive distribution into the distal stomach as emptying progresses. In a group of dyspeptic patients, impaired accommodation of the proximal stomach to a meal with increased distribution to the distal stomach was demonstrated..$^{2}$ Impaired accommodation of the proximal stomach is associated with dyspeptic symptoms, especially early satiety and weight loss. ${ }^{4}$ However, it is still not known which region of the stomach is responsible for symptom generation in impaired gastric accommodation.

Previous studies in animals have shown that antral distension, unlike fundic distension, induces vomiting and that vagal afferent discharges differ between the antrum and fundus. ${ }^{5}{ }^{6}$ To date, human studies of the differential effects of proximal and distal gastric distension on induction of symptoms have shown conflicting results, ${ }^{7-9}$ which is at least partly attributable to technical and methodological differences. On the other hand, simultaneously applied low level stimuli in the gut were found to increase the mechanosensitivity of the human gut. ${ }^{10-12}$ At present, it is not known whether simultaneously applied low level distending stimuli in the stomach has such a modulatory role.

Thus in the present study, we aimed to define differences in the sensorimotor response to distension between proximal and distal gastric distension in normal human subjects, and to determine the effects of simultaneously applied

low level gastric distension on gastric tone and mechanosensitivity.

\section{MATERIALS AND METHODS \\ Subjects}

Fourteen healthy volunteers ( 12 men and two women; mean age 28.9 (1.3) years (range 23-36)) participated in the study. None of the subjects had symptoms or a history of gastrointestinal disease or drug allergies, nor were they taking any medications. Written informed consent was obtained from each participant and the study protocol was approved by the ethics committee of the University Hospital.

\section{Techniques of dual barostat study}

Following an overnight fast of at least 12 hours, a polyvinyl tube (Salem sump tube $14 \mathrm{Ch}$; Sherwood Medical, Petit Rechain, Belgium) with two plastic bags attached (proximal one $1200 \mathrm{ml}$ capacity, $12 \mathrm{~cm}$ maximal diameter; distal one $900 \mathrm{ml}, 10 \mathrm{~cm}$ maximal diameter) at a distance of $2 \mathrm{~cm}$ between both bags, was introduced through the mouth and secured to the subject's chin with adhesive tape. The $10 \mathrm{~cm}$ long distal tip of the tube was fluoroscopically positioned through the pylorus, ensuring adequate location of the bags in the proximal and distal stomach (fig 1). At the beginning and end of each study, the position of the bags was verified fluoroscopically.

The polyvinyl tube was then connected to two different computer driven programmable volume displacement barostat devices (Synectics Visceral Stimulator, Stockholm, Sweden, and Distender Series II, Toronto, Canada). The

Abbreviations: MDP, minimal distending pressure; VAS, visual analogue scale 


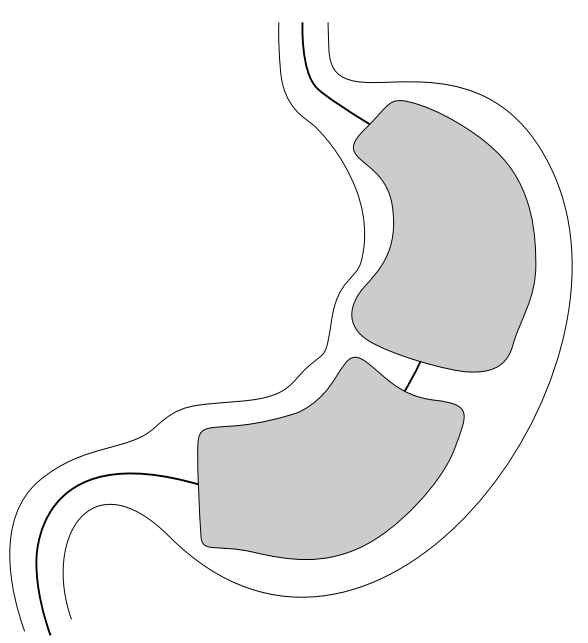

Figure 1 Position of the barostat tube and two bags in the stomach. The $10 \mathrm{~cm}$ long distal tip of the barostat tube was positioned over the pylorus and two adherent bags were located in the proximal and distal stomach.

barostat device can deliver volume ramps or pressure steps at different rates, while simultaneously monitoring pressure and volume at a sampling rate of eight samples per second. To unfold each intragastric bag, it was inflated with a fixed volume of $300 \mathrm{ml}$ of air for two minutes with the study subject in a recumbent position, and again deflated completely. After a 10 minute equilibration period, subjects were positioned in a comfortable sitting position with the knees slightly bent $\left(80^{\circ}\right)$, in a bed specifically designed for that purpose.

\section{Sensitivity study}

After a 30 minute accommodation period, minimal intragastric distending pressure (MDP) for both barostat bags was first determined as the lowest pressure level that provided an intrabag volume of $30 \mathrm{ml}$ or more. ${ }^{12}$ This pressure level equilibrates intra-abdominal pressure. With subjects in the sitting position, MDP was determined by increasing intrabag pressure by $1 \mathrm{~mm} \mathrm{Hg}$ every three minutes. Subsequently, stepwise isobaric distensions of either bag (pressure sensitivity test) were performed with and without simultaneous background distension of the other bag at a pressure of $\mathrm{MDP}+2 \mathrm{~mm} \mathrm{Hg}$, in a randomised manner. Consequently, four sets of pressure sensitivity tests (proximal and distal, with and without background distension of the other bag) were randomly accomplished in each subject (fig 2).

Each set of sequential isobaric distensions was performed in stepwise increments of $2 \mathrm{~mm} \mathrm{Hg}$, starting from MDP, and each lasting for two minutes, while the corresponding intragastric volume was recorded. Subjects were instructed to score their perception of upper abdominal sensations at the end of every distending step, using both a graphic rating scale that combined verbal descriptors on a scale graded $0-6^{12}$ and $100 \mathrm{~mm}$ visual analogue scales (VAS) for seven dyspeptic symptoms (epigastric discomfort, fullness, bloating, nausea, belching, epigastric burning, and satiety).

\section{Accommodation study}

After sensitivity testing, both bags were simultaneously inflated at a pressure of $\mathrm{MDP}+2 \mathrm{~mm} \mathrm{Hg}$, and intrabag volume of the proximal stomach was recorded over 20 minutes before a mixed liquid meal $(200 \mathrm{ml}, 300 \mathrm{kcal}, 13 \%$ proteins, $48 \%$ carbohydrates, 39\% lipids; Nutridrink, Nutricia, Belgium) and then 40 minutes after the meal (fig 2). For comparison, all subjects underwent a conventional single barostat study on a separate day to measure proximal gastric

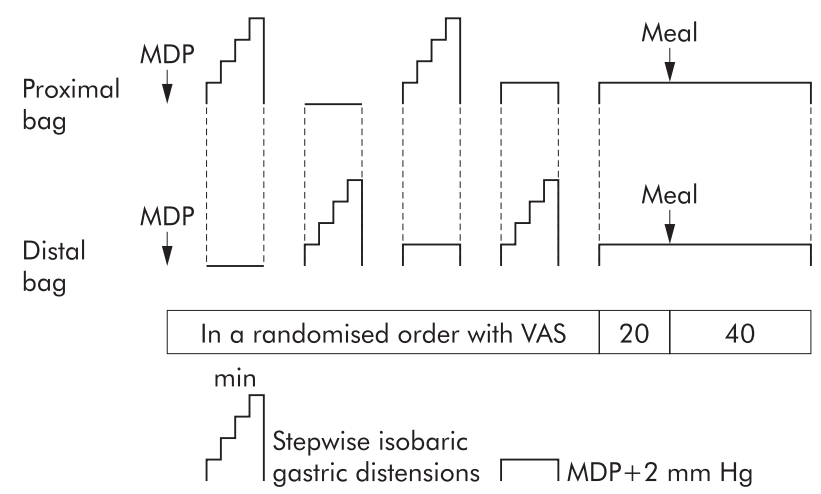

Figure 2 Study protocol. Pressure sensitivity tests with either bag were performed with and without simultaneous distension of the other bag at minimal distending pressure (MDP) $+2 \mathrm{~mm} \mathrm{Hg}$, in a randomised manner. Afterwards, both bags were simultaneously inflated at MDP $2 \mathrm{~mm} \mathrm{Hg}$ and proximal gastric accommodation to a meal was measured. VAS, visual analogue scale.

accommodation to the same meal using a conventional single barostat bag.

\section{Data analysis}

For each two minute distending period, the dependent variable was calculated by averaging the recordings. The thresholds for first perception and discomfort were computed after the experiments by analysing the perception score corresponding to each distension step. Perception threshold was defined as the first level of pressure that had evoked a perception score of 1 or more during isobaric distensions. Discomfort threshold was defined as the first level of pressure that provoked a perception score of 5 or more during isobaric distensions. Pressure thresholds were expressed as pressures relative to MDP. Gastric wall tension at the threshold for first perception and for discomfort was calculated using Laplace's law. ${ }^{13}$ The use of Laplace's law requires a number of assumptions, a spherical shape of the distended organ segment, which are not fulfilled. ${ }^{14}$ However, a previous analysis by Notivol et al suggested that the margin of error that occurs with deviation from a spherical shape is acceptable. ${ }^{13}$ Gastric compliance was measured as the linear slope obtained from the steep part $(2 \sim 12 \mathrm{~mm} \mathrm{Hg}$ above MDP) of the pressure-volume curve.

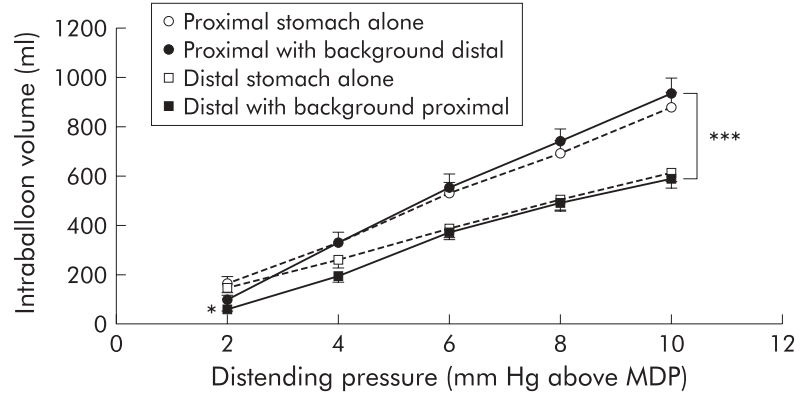

Figure 3 Pressure-volume curve. Compliance of the distal stomach was significantly lower than that of the proximal stomach $\left(^{* * *} p<0.001\right.$, paired $t$ test). Simultaneous low level distending stimuli of the proximal stomach decreased distal intraballoon volume at minimal distending pressure (MDP) $+2 \mathrm{~mm} \mathrm{Hg}$ ( ${ }^{*} \mathrm{p}<0.05$, paired $t$ test). This was associated with an increased slope of the pressure-volume relationship in the lower but not the higher distending range $(n=13$ for each data point at pressures of 2, 4, and $6 \mathrm{~mm} \mathrm{Hg}$ above MDP; $\mathrm{n}=12$ or 13 at $8 \mathrm{~mm} \mathrm{Hg}$ above MDP; and $\mathrm{n}=8-11$ at $10 \mathrm{~mm} \mathrm{Hg}$ above MDP). 
Gastric tone was measured by calculation of the mean intrabag volume for consecutive five minute intervals during the long distending periods at a pressure of $\mathrm{MDP}+2 \mathrm{~mm} \mathrm{Hg}$. Meal induced gastric relaxation was quantified by calculating the difference between the average intrabag volume over five minutes just before and the first 40 minutes after administration of the meal. The maximum postprandial volume increase and the time needed to reach the maximum postprandial volume were assessed. In addition, duration of meal induced relaxation, defined as the time needed before the intrabag volume was again at or below the preprandial volume just before a meal, was also determined.

\section{Statistical analysis}

Thresholds for first perception and discomfort and compliance, obtained in the pressure-volume curve, were compared by paired $t$ test. Analysis of variance for repeated measures was used for comparison of the first three steps in the pressure-perception curve, which all of the subjects had taken. The area under the pressure-perception curve and the pressure-symptom curve for the same pressure steps was compared by paired $t$ test, in which the first 4.8 steps, on average (range 3-8 steps), could be analysed. Change in intrabag volumes after ingestion of a meal was evaluated using analysis of variance for repeated measures. In addition, a paired $t$ test was used for comparison of meal induced gastric relaxation, maximum postprandial volume increase, and its occurrence time from the meal between dual and single barostat bag studies. A p value of $<0.05$ was considered statistically significant. Data are presented as mean (SEM).

\section{RESULTS}

\section{Conduct of the study}

Oral intubation with subsequent positioning of the barostat bags in the proximal and distal stomach was well tolerated by all subjects. A metallic stiffening guidewire, introduced into the inflation channel of the distal balloon, was used as an aid to overcome the narrow passage in the throat and the upper oesophageal sphincter, and was removed when both bags reached the stomach. Fluoroscopic control before and after the study confirmed maintenance of the exact location of the barostat bags in the stomach in all subjects. Migration of the distal bag beyond the pylorus into the duodenum was never observed. At the end of the study, the assembly was easily removed by gentle traction after deflation of both bags. All recordings were technically adequate and could be used for analysis.

Mean MDP was $7.6(0.4) \mathrm{mm} \mathrm{Hg}$ in the proximal stomach and $7.6(0.4) \mathrm{mm} \mathrm{Hg}$ in the distal stomach (NS). Pressure sensitivity tests without simultaneous distension were first performed in seven subjects, and those with simultaneous distension were first performed in the other subjects. Likewise, proximal gastric mechanosensitivity was first

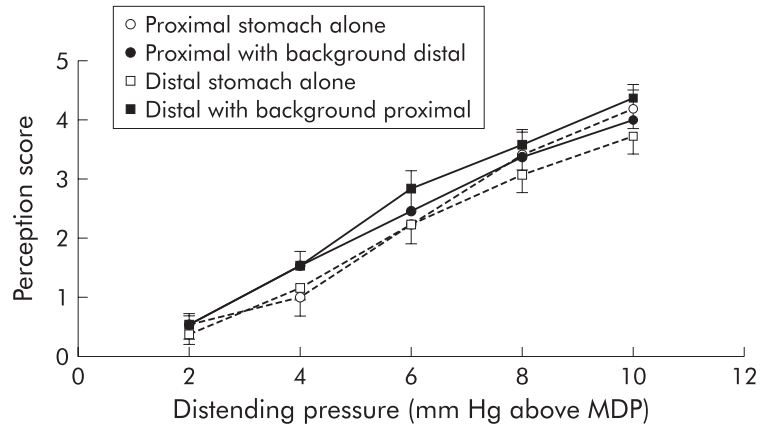

Figure 4 Pressure-perception curve. Perception scores at the same distending pressures did not differ between the proximal and distal stomach. Pressure-perception curves remained unaltered during simultaneous low level distending stimuli (ANOVA for repeated measures) ( $n=13$ for each data point at pressures of 2,4 , and $6 \mathrm{~mm} \mathrm{Hg}$ above minimal distending pressure (MDP); $n=12$ or 13 at $8 \mathrm{~mm} \mathrm{Hg}$ above MDP; and $n=8-11$ at $10 \mathrm{~mm} \mathrm{Hg}$ above MDP).

Table 2 Comparison of the area under the pressuresymptom curve for individual dyspeptic symptoms between the proximal and distal stomach (paired $t$ test)

\begin{tabular}{llr}
\hline & \multicolumn{2}{l}{ Area under the curve $(\mathbf{m m} \times \mathbf{m m ~ H g})$} \\
\cline { 2 - 3 } & Proximal stomach & Distal stomach \\
\hline Epigastric discomfort & $24.6(2.7)$ & $24.0(4.4)$ \\
Fullness & $25.6(2.8)$ & $28.0(4.3)$ \\
Bloating & $28.8(2.9)$ & $32.4(4.5)$ \\
Nausea & $5.2(1.0)$ & $5.2(1.0)$ \\
Belching & $10.2(1.7)$ & $8.8(1.6)$ \\
Epigastric burning & $13.8(2.3)$ & $20.0(3.9)$ \\
Satiety & $17.2(2.7)$ & $22.6(4.2)$ \\
\hline \multirow{2}{*}{ Data are given as mean (SEM). }
\end{tabular}

measured in seven subjects, and distal gastric mechanosensitivity was first measured in the other subjects.

\section{Compliance}

Compliance of the distal stomach was significantly lower than that of the proximal stomach (53.0 (3.9) $v 90.7$ (5.5) ml/ $\mathrm{mm} \mathrm{Hg} ; \mathrm{p}<0.001)$. Low level distal gastric distension did not influence compliance of the proximal stomach (90.7 (5.5) $\mathrm{v}$ 94.5 (6.2) ml/mm Hg; NS) (fig 3). Low level proximal gastric distension significantly decreased intraballoon volume at $\mathrm{MDP}+2 \mathrm{~mm} \mathrm{Hg}(\mathrm{p}<0.05)$, and this was associated with an increase in the slope of the pressure-volume relationship for lower levels of distension (2-6 mm Hg above MDP: 60.0 (6.7) $v 76.9(7.5) \mathrm{ml} / \mathrm{mm} \mathrm{Hg} ; \mathrm{p}=0.01)$ but not for the steep part of the curve (6-12 mm Hg above MDP: 52.0 (5.5) v 52.1 (6.3) $\mathrm{ml} / \mathrm{mm} \mathrm{Hg}$; NS).

Table 1 Sensitivity to isobaric distensions between the proximal and distal stomach and influence of simultaneous low level gastric distension

\begin{tabular}{|c|c|c|c|c|c|c|}
\hline & \multicolumn{3}{|c|}{ Threshold for first perception } & \multicolumn{3}{|c|}{ Threshold for discomfort } \\
\hline & $\begin{array}{l}\text { Pressure } \\
\text { (mm Hg above MDP) }\end{array}$ & $\begin{array}{l}\text { Volume } \\
\text { (ml) }\end{array}$ & $\begin{array}{l}\text { Tension } \\
(\mathbf{c m} \times \mathbf{m m ~ H g})\end{array}$ & $\begin{array}{l}\text { Pressure } \\
\text { (mm Hg above MDP) }\end{array}$ & $\begin{array}{l}\text { Volume } \\
\text { (ml) }\end{array}$ & $\begin{array}{l}\text { Tension } \\
(\mathbf{c m} \times \mathbf{m m ~ H g})\end{array}$ \\
\hline Proximal stomach & $4.0(0.6)$ & $336(56)$ & $20.2(3.3)$ & $10.9(0.8)$ & $916(70)$ & $87.0(8.7)$ \\
\hline Distal stomach & $3.9(0.6)$ & $262(57)$ & $19.1(4.2)$ & $12.1(1.0)$ & $625(58)^{* *}$ & $85.2(9.1)$ \\
\hline Proximal stomach* & $3.1(0.4)$ & $286(60)$ & $15.9(2.6)$ & $10.1(0.7)$ & $852(65)$ & $78.4(7.3)$ \\
\hline Distal stomach* & $2.9(0.3)$ & $142(33)$ & $12.6(2.1)$ & $11.4(1.0)$ & $613(47)^{\star *}$ & $79.0(8.4)$ \\
\hline
\end{tabular}

Data are given as mean (SEM).

*With simultaneous low level gastric distension.

${ }^{* *} \mathrm{p}<0.001$ between the proximal and distal stomach (paired $t$ test). 

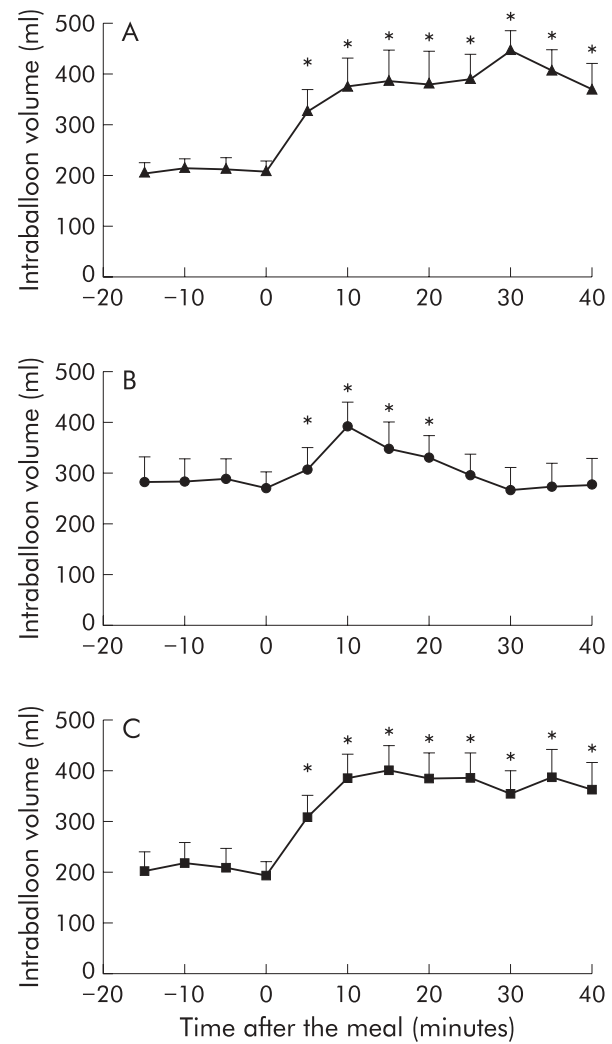

Figure 5 Meal induced accommodation. In the conventional single barostat bag studies, significant proximal gastric relaxation was continuous during the first 40 minutes after the meal (A). In the dual barostat bag studies, this significant postprandial relaxation of the proximal stomach was observed only during the first 25 minutes after the meal. (B). The barostat bag in the distal stomach also displayed significant relaxation in response to a meal (C) (* $p<0.05$, ANOVA for repeated measures).

\section{Mechanosensitivity}

During stepwise isobaric distensions, no significant differences in pressures, corresponding volumes, or corresponding wall tensions inducing first perception were observed between the proximal and distal stomach. At the thresholds for discomfort, the corresponding volumes of the distal stomach were significantly lower than those of the proximal stomach, but pressures and corresponding wall tensions did not differ between the proximal and distal stomach (table 1).

In keeping with these results, perception scores at the same distending pressures did not differ between the proximal and distal stomach (fig 4). Similarly, no significant differences in the area under the pressure-symptom curve for individual symptoms such as epigastric discomfort, fullness, bloating, nausea, belching, epigastric burning, or satiety were observed between the proximal and distal stomach (table 2).

\section{Effect of low level distension on mechanosensitivity}

Low level distending stimuli of $\mathrm{MDP}+2 \mathrm{~mm} \mathrm{Hg}$ either in the proximal stomach or in the distal stomach were perceived in six subjects $(6 / 14(43 \%))$ whose perception scores were all 1 (vague perception). Such stimuli did not influence pressures, corresponding volumes, or corresponding wall tensions at the thresholds for first perception, both in the proximal stomach and in the distal stomach. Similarly, pressures, corresponding volumes, and corresponding wall tensions at the thresholds for discomfort in the proximal and distal stomach were not significantly affected by simultaneously applied low level gastric distension (table 1).

\section{Effect of low level distension on postprandial relaxation}

Pre-meal baseline values were stable, showing adequate recovery after stepwise distensions (fig 5). In the conventional single barostat bag studies, intrabag volume of the proximal stomach was significantly increased during the first 40 minutes after administration of the meal (fig 5A). However, with the distal bag simultaneously inflated at a pressure of $\mathrm{MDP}+2 \mathrm{~mm} \mathrm{Hg}$, a significant postprandial increase in proximal gastric bag volume was observed only during the first 25 minutes after administration of the meal (fig 5B). Similarly, duration of meal induced relaxation of the proximal stomach in the dual barostat bag studies was shorter than 40 minutes, the total postprandial period in the present study, in nine $(9 / 14(64 \%))$ subjects, while it was longer than 40 minutes in all cases when gastric accommodation was measured using a conventional single barostat bag. Actually, low level distal gastric distension significantly decreased postprandial relaxation of the proximal stomach (54.2 (28.4) $v 176.0(34.3) \mathrm{ml} ; \mathrm{p}<0.01)$ and the maximum postprandial volume increase (173.5 (30.1) v 266.8 (31.5) ml; $\mathrm{p}<0.05)$ compared with the conventional single barostat bag studies. However, the occurrence time of maximum postprandial relaxation from the meal (16.4 (2.8) $v 22.1$ (2.2) minutes; NS) did not differ significantly.

Mean intra-balloon volume in the distal stomach bag increased from 207 (20) before the meal to 371 (19) $\mathrm{ml}$ postprandially $(\mathrm{p}<0.02)$. A significant increase in intraballoon volume over preprandial volume occurred as early as five minutes after the meal and was maintained until the end of the measurement, 40 minutes postprandially (all $\mathrm{p}<0.05$ ). The maximum volume increase of 231 (28) $\mathrm{ml}$ over basal volume was reached 19 (3) minutes after meal ingestion (fig 5C).

\section{DISCUSSION}

Differences in the properties and functions between the proximal and distal stomach have been recognised in human studies as well as in animals. In dogs, distension of pyloric pouches invariably produced vomiting, which was completely abolished by vagotomy. In contrast, vomiting could not be induced by comparable distension of fundic pouches. ${ }^{5}$ In the ferret, vagal afferent discharges induced by antral distension were correlated with enhanced antral contractions whereas those induced by fundic distension were unrelated to intragastric pressure. ${ }^{6}$ These animal studies suggest that symptom profiles induced by gastric distension may differ between proximal and distal gastric distension. However, this possibility has not yet been fully investigated in humans, which is at least partly attributable to the technical and methodological difficulties and the diversity and complexity of human symptoms.

A previous study in healthy humans, using a conventional single barostat bag, showed that nausea was induced by distal gastric distension, but not by proximal gastric distension. ${ }^{7}$ For distal distension, the bag, the capacity of which was $500 \mathrm{ml}$, was advanced to the antrum and its position in the distal stomach was confirmed by fluoroscopy. In that study, bloating and epigastric pain were developed at lower pressures in the distal stomach compared with the proximal stomach. ${ }^{7}$ These findings suggest that distal gastric distension is more liable to genesis of symptoms than proximal gastric distension, possibly related to regional differences in the sensitivities of gastric mechanoreceptors. However, another human study, using ultrasonography, showed contrasting results. Bloating, fullness, epigastric pain, and heartburn were significantly and directly correlated not only with distal gastric distension but also with proximal gastric distension. ${ }^{8}$ In that study, functional dyspepsia patients 
represented a higher symptom score for smaller values of proximal gastric distension and greater values of distal gastric distension, compared with normal subjects. In addition, the correlation coefficient between the score of bloating and fullness and the size of the gastric measurement in dyspeptics was significantly different from normal controls for proximal gastric measurement but not for distal gastric measurement. ${ }^{8}$ These observations imply that the proximal stomach may play a major role in symptom production in patients with functional dyspepsia. Thus previous human studies have shown conflicting results in this area.

Gastric barostat has been regarded as the gold standard for assessment of gastric sensitivity to distensions. However, unlike the proximal stomach, it is not easy to measure distal gastric sensitivity to isobaric distensions. In recent studies, a bag filled with water, instead of an air filled barostat bag, was used for distal gastric distension, ${ }^{10}$ or a conventional barostat bag was positioned in the distal stomach. ${ }^{7}$ However, in the latter case, once the distal gastric bag is inflated by air, it is liable to proximal migration. Because maintenance of a barostat bag in the same gastric region during the study is essential for the reliability of the data, we designed a long tube with two adherent barostat bags. In order to position two barostat bags in adequate places and prevent proximal migration of the distal bag, the two bags are separated by a small distance and the tube has a $10 \mathrm{~cm}$ long distal tip positioned over the pylorus. In fact, maintenance of the correct location of two barostat bags was confirmed fluoroscopically before and after the study.

The proximal stomach can accommodate food through meal induced relaxation. Insufficient relaxation of the proximal stomach during and after ingestion of a meal may increase pressures and activate mechanoreceptors, resulting in dyspeptic symptoms. On the other hand, failure of the proximal stomach to accommodate properly may force the meal into the distal stomach which becomes overdistended. Several studies have suggested that the distal stomach is more responsible for symptomatic gastric dysaccommodation in functional dyspepsia patients and in normal subjects. ${ }^{9} 1516$ The results of the present study showed a regional difference in compliance of the gastric wall. The distal gastric wall was less compliant compared with the proximal gastric wall. Corresponding volumes at thresholds for discomfort were significantly lower in the distal stomach than in the proximal stomach but corresponding wall tensions were not different. Furthermore, symptom profiles induced by gastric distension did not differ between proximal and distal gastric distension. These findings imply that the distal stomach may produce greater symptoms in response to the same volume of distension, due to lesser compliance of the distal stomach. Considering that gastric wall tension determines perception of gastric distension, ${ }^{17}$ the sensitivity of mechanoreceptors does not seem to be regionally different in normal healthy subjects. Also, the results of the present study showed that symptoms induced by gastric distension were not region specific in healthy subjects. The findings of the present study do not necessarily apply to patients with functional dyspepsia. Firstly, the majority of healthy volunteers in the present study were men whereas pathophysiological studies in functional dyspepsia investigated mostly women. Secondly, normal sensory pathways and mechanisms may be altered profoundly under pathophysiological circumstances, such as visceral hypersensitivity or longstanding dyspeptic symptoms.

Previous studies have shown that perception of simultaneously applied mechanical stimuli in the gut is enhanced by a phenomenon of spatial summation. ${ }^{10} 11$ Low unperceived stimulation was also found to play a modulatory role in gut perception. ${ }^{12}$ Non-specific stimulation of intestinal afferents by low unperceived stimuli exerted a sensitising effect and increased perception of well tolerated intestinal distensions up to levels of discomfort. In contrast with perceived distending stimuli showing a phenomenon of spatial summation, unperceived distending stimuli were reported to have no additive effects in the perception of gut distension. ${ }^{12}$ However, it is not known whether simultaneously applied low level gastric distension may have a modulatory role in sensory circuits of the human stomach. In the present study, simultaneous gastric distension at a pressure of $2 \mathrm{~mm} \mathrm{Hg}$ above MDP, which was minimally perceived in only six subjects $(43 \%)$, did not influence the sensitivity to gastric distension, indicating that such stimuli do not produce summative effects in the perception of gastric distension. This finding does not exclude summative effects of higher levels of gastric distension.

Previous studies have revealed a role of enterogastric reflexes in the control of gastric tone. ${ }^{18-20}$ Gastrogastric relaxatory reflexes are also known to play a role during the initial accommodation process, before nutrients enter the intestine. ${ }^{20}$ However, gastrogastric reflexes have not been fully investigated to date because these studies, especially in humans, have been hampered by the methodological difficulties of measuring tone when stimuli are concomitantly applied in the stomach. ${ }^{21}$ It is unclear whether a distending pressure of $2 \mathrm{~mm} \mathrm{Hg}$ above MDP may induce gastrogastric reflexes, hereby affecting gastric tone. A recent study, using a barostat in the proximal stomach and ultrasonography of the distal stomach, showed that a pressure of $1 \mathrm{~mm} \mathrm{Hg}$ above MDP in the gastric fundus had no effect on fasting antral size but caused further dilatation of the antrum after a meal. This was explained by displacement of the meal towards the distal stomach because of the dilated proximal barostat bag after a meal. ${ }^{22}$ In the present study, we observed that low level proximal gastric distension induces lower volumes in the distal stomach to low level distending pressures. It is unclear whether this represents a true gastrogastric reflex pathway or whether it is a passive effect of the proximal stomach balloon influencing expansion of the distal balloon. In contrast with the significant effect on distal gastric compliance, our results showed that such background distending stimuli in the distal stomach did not affect proximal gastric compliance.

Using a dual barostat bag assembly, we were able to demonstrate that not only the proximal stomach, but also the distal stomach, displays a marked relaxatory response to meal ingestion. However, postprandial relaxation of the proximal stomach and its duration were decreased under simultaneously applied low level distending stimuli in the distal stomach. This finding may be explained in a number of ways. Delivery of the meal to the duodenum may be hampered by the dilated barostat bags positioned in the proximal and distal stomach. However, a modulatory role of low level distal gastric distension in postprandial proximal gastric tone is also possible. Finally, a passive effect of a distended distal balloon occupying part of the proximal stomach also cannot be excluded. As the mechanisms underlying gastric accommodation to a meal and gastrogastric reflexes have not been fully elucidated, further investigation is required to determine the most likely explanation.

In conclusion, compared with the proximal stomach, the distal stomach is less compliant but its mechanosensitivity is not different. Symptoms induced by gastric distension are not region specific. Simultaneously applied low level gastric distension of one segment does not affect gastric mechanosensitivity of the other segment. Both the proximal and distal stomach relax after ingestion of a meal. Meal induced relaxation occurs both in the proximal and distal stomach, 
but simultaneous low level antral distension decreases proximal gastric accommodation.

\section{Authors' affiliations}

K-J Lee, R Vos, J Janssens, J Tack, Division of Gastroenterology, Department of Internal Medicine, University Hospital Gasthuisberg, University of Leuven, Belgium

\section{REFERENCES}

1 Read NW, Houghton LA. Physiology of gastric emptying and pathophysiology of gastroparesis. Gastroenterol Clin North Am 1989;11:625-30.

2 Troncon LEA, Bennett RJM, Ahluwalia NK, et al. Abnormal intragastric distribution of food during gastric emptying in functional dyspepsia patients. Gut 1994:35:327-32.

3 Gilja $\mathrm{OH}$, Hausken T, Wilhelmsen I, et al. Impaired accommodation of proximal stomach to a meal in functional dyspepsia. Dig Dis Sci 1996;41:689-96

4 Tack J, Piessevaux H, Coulie B, et al. Role of impaired gastric accommodation to a meal in functional dyspepsia. Gastroenterology 1998:115:1346-52.

5 Goldberg SL. The afferent paths of nerves involved in the vomiting reflex induced by distension of an isolated pyloric pouch. Am J Physiol 1931;99:156-9.

6 Andrews PLR, Grundy D, Scratcherd T. Vagal afferent discharge from mechanoreceptors in different regions of the ferret stomach. J Physiol (Lond) 1980;298:513-24.

7 Ladabaum URI, Koshy SS, Woods ML, et al. Differential symptomatic and electrogastrographic effects of distal and proximal human gastric distension. Am J Physiol 1998;275:G418-24.

8 Marzio L, Falcucci M, Grossi L, et al. Proximal and distal gastric distension in normal subjects and $\mathrm{H}$. pylori-positive and -negative dyspeptic patients and correlation with symptoms. Dig Dis Sci 1998;43:2757-63.
9 Caldarella MP, Azpiroz F, Malagelada JR. The distal stomach is responsible for symptomatic gastric accommodation in functional dyspepsia. Gastroenterology 1999;118:A670.

10 Serra J, Azpiroz F, Malagelada JR. Perception and reflex responses to intestinal distension and modified by simultaneous or previous stimulation. Gastroenterology 1995;109:1742-9.

11 Serra J, Azpiroz F, Malagelada JR. Modulation of gut perception by spatial summation phenomena. J Physiol (Lond) 1998;506:579-87.

12 Accarino AM, Azpiroz F, Malagelada JR. Gut perception in humans is modulated by interacting gut stimuli. Am J Physiol 2002;282:G220-5.

13 Notivol R, Coffin B, Azpiroz F, et al. Gastric tone determines the sensitivity of the stomach to distention. Gastroenterology 1995;108:330-6.

14 Gregersen H, Kassab G. Biomechanics of the gastrointestinal tract. Neurogastroenterol Motil 1996:8:277-97.

15 Boeckxstaens GE, Hirsch DP, Kuiken SD, et al. The proximal stomach and postprandial symptoms in functional dyspeptics. Am J Gastroenterol 2002;97:40-8.

16 Jones KL, Doran SM, Hveem K, et al. Relation between postprandial satiation and antral area in normal subjects. Am J Clin Nutr 1997:66:127-32.

17 Distrutti E, Azpiroz F, Soldevilla A, et al. Gastric wall tension determines perception of gastric distension. Gastroenterology 1999;116:1035-42.

18 Azpiroz F, Malagelada JR. Intestinal control of gastric tone. Am J Physiol 1985:249:G501-9.

19 Mayer EA. The physiology of gastric storage and emptying. In: Johnson LR, eds. Physiology of the gastrointestinal tract, 3rd edn. New York: Raven, 1994:929-76.

20 Feinle C, D'Amato M, Read NW. Effects of duodenal nutrients on sensory and motor responses of the human stomach to distension. Am J Physiol 1997; 273:G721-6.

21 Villanova N, Azpiroz F, Malagelada JR. Gastrogastric reflexes regulating gastric tone and their relationship to perception. Am J Physiol 1997:273:G464-9.

22 Mundt MW, Hausken T, Samsom M. Effect of intragastric barostat bag on proximal and distal gastric accommodation in response to liquid meal. Am J Physiol 2002;283:G681-6.

\section{EDITOR'S QUIZ: GI SNAPSHOT}

\section{Answer}

From question on page 937

The computed tomography (CT) scan showed a high signal intensity mass over the hilum of the liver compatible with aneurysmal dilatation of the portal vein. The biliary system, hepatic parenchyma, and spleen were normal (fig 1).

An extrahepatic portal vein aneurysm was confirmed in the spiral $\mathrm{CT}$ angiography as a homogeneous contrast enhancing lesion near the junction of the superior mesenteric vein and the splenic vein. In this case, a conservative management strategy was adopted, with regular review of the size of the aneurysm. At present, she is healthy and has developed no complications.

Portal vein aneurysm is a very rare entity with no more than 50 cases published. Despite the fact that the postulated origins seem to be controversial, its relation to portal hypertension has been emphasized. Although there is no consensus on treatment, management includes careful follow up in asymptomatic patients without underlying liver disease or portal hypertension. 